\title{
A NOTE ON MAPPINGS AND ALMOST MENGER AND RELATED SPACES
}

\section{Ljubiša Kočinac $^{1 *}$}

${ }^{1}$ Faculty of Sciences and Mathematics, University of Niš, Niš, Serbia.

\section{ABSTRACT \\ We consider behaviour of the almost Menger, almost Rothberger, almost Hurewicz, and almost $\gamma$ - \\ set properties under several kinds of mappings between topological spaces.}

Key words: Almost Menger, almost Hurewicz, almost Rothberger, almost $\gamma$-set, $\Theta$-continuity, weak continuity.

\section{INTRODUCTION}

Throughout the paper $(X, \tau)$, or shortly $X$, will denote a topological space on which no separation axioms are assumed unless explicitly stated. For a subset $A$ of $(X, \tau), \operatorname{Int}(A)$ and $\operatorname{Cl}(A)$ will denote the interior and the closure of $A$, respectively. Our other notation and terminology are standard as in (Engelking, 1989).

The following classical covering properties were defined long time ago. A topological space $X$ is said to be:

(1) A Menger space (or has the Menger (covering) property) (Menger,1924), (Hurewicz, 1925) if for each sequence $\left(\mathcal{U}_{n}\right)_{n \in N}$ of open covers of $X$ there exists a sequence $\left(\mathcal{V}_{\mathrm{n}}\right)_{n \in N}$ of finite sets such that for each $n \in N, \quad \mathcal{V}_{n}$ is a subset of $\mathcal{U}_{n}$ and $\bigcup_{n \in N} \mathcal{V}_{n}$ is an open cover of $X$;

(2) A Hurewicz space (Hurewicz, 1925) if for each sequence $\left(\mathcal{U}_{n}\right)_{n \in N}$ of open covers of $X$ there is a sequence $\left(\mathcal{V}_{n}\right)_{n \in N}$ such that for each $\mathrm{n}, \mathcal{V}_{n}$ is a finite subset of $\mathcal{U}_{n}$ and each $x \in X$ belongs to $\mathrm{U} \mathcal{V}_{n}$ for all but finitely many $n$;

(3) A Rothberger space (Rothberger, 1938) if for each sequence $\left(\mathcal{U}_{n}\right)_{n \in N}$ of open covers of $X$ there exists a sequence $\left(U_{n}\right)_{n \in N}$ such that for each $n \in N, U_{n}$ $\epsilon \mathcal{U}_{n}$ and $\left\{U_{n}: n \epsilon N\right\}$ is an open cover of $X$;

(4) A $\gamma$-set (Gerlits \& Nagy, 1982) if for each sequence $\left(\mathcal{U}_{n}\right)_{n \in N}$ of $\omega$-covers of $X$ there is a sequence $\left(U_{n}\right)_{n \in N}$ such that for each $n \epsilon N, U_{n} \epsilon \mathcal{U}_{n}$ and each $x \epsilon$ $X$ belongs to $U_{n}$ for all but finitely many $n$.

[Recall that an open cover $\mathcal{U}$ of a space $X$ is called an $\omega$-cover if $X$ does not belong to $\mathcal{U}$ and each finite subset of $X$ is contained in a member of $\mathcal{U}$.]
For more information about these spaces we refer the reader, for example, to (Kočinac, 2006), (Kočinac, 2015). Note that the first two properties are between two important covering properties -- $\sigma$-compactness and Lindelöfness. In the last two decades these spaces and their relations with other mathematical disciplines (such as game theory, Ramsey theory and so on) have been systematically studied by a big number of mathematicians. Also, some weaker forms of these properties were investigated from different points of view, see (Babinkostova et. al., 2012), (Babinkostova et. al., 2013), (Daniels, 1988), (Di Maio \& Kocinac, 2008), (Kocev, 2009), (Kočinac, 1999), (Kočinac, 2001), (Pansera, 2012), (Sabah et al., 2016), (Sakai, 2013).

In (Kočinac, 1999), Kočinac introduced almost Menger and almost Rothberger spaces as generalizations of almost Lindelöf spaces introduced in (Willard, \& Dissanayake, 1984). In (Di Maio \& Kocinac, 2008) the almost Menger property was considered in hyperspaces. Further investigation of almost Menger spaces was done in (Kocev, 2009), and (Babinkostova et. al., 2012), (Babinkostova et. al., 2013). In (Kocev, 2009), the almost $\gamma$-sets have been introduced.

Definition 1.1. (a) (Kočinac, 1999) A space $(X, \tau)$ is almost Menger if for each sequence $\left(\mathcal{U}_{\mathrm{n}}\right)_{n \in N}$ of open covers of $X$ there is a sequence $\left(\mathcal{V}_{n}\right)_{n \in N}$ of finite sets such that for each $n \in N, \mathcal{V}_{\mathrm{n}}$ is a finite subset of $\mathcal{U}_{n}$ and $\mathrm{U}_{n \in N} \mathrm{U}\left\{\mathrm{Cl}(V): V \in \mathcal{V}_{n}\right\}=X$;

(b) (Kočinac, 1999) A space $X$ is almost Rothberger if for each sequence $\left(\mathcal{U}_{\mathrm{n}}\right)_{\mathrm{n} \in \mathbf{N}}$ of open covers of $X$ there is a sequence $\left(U_{n}\right)_{n \in N}$ such that for each $n \in N, \mathrm{U}_{\mathrm{n}} \in \mathcal{U}_{\mathrm{n}}$ and $\mathrm{U}_{n \in N} \mathrm{Cl}\left(U_{n}\right)=X$; 
(c) (Kocev, 2009) A space $X$ is an almost $\gamma$-set if for each sequence $\left(\mathcal{U}_{\mathrm{n}}\right)_{\mathrm{n} \in \mathrm{N}}$ of $\omega$-covers of $X$ there exists a sequence $\left(U_{n}\right)_{n \in N}$ such that for each $n \in N, U_{n}$ $\epsilon \mathcal{U}_{n}$ and each $x \in X$ belongs to $\mathrm{Cl}\left(U_{n}\right)$ for all but finitely many $n$.

In this paper we introduce the class of almost Hurewicz spaces and consider behaviour of the almost Menger, almost Hurewicz, almost Rothberger and almost $\gamma$-set properties under several kinds of mappings between topological spaces. Note that these properties are useful in studying of non-regular spaces.

\section{RESULTS}

We recall now the notion of (strong) $\theta$-continuity which is an important slight generalization of continuity.

A mapping $f: X \rightarrow Y$ is $\theta$-continuous (Fomin, 1941), (Fomin, 1943), see also (Pansera, 2012) (strongly $\theta$-continuous) (Long \& Herrington, 1981) if for each $x \in X$, and each open set $V$ in $Y$ containing $f(x)$ there is an open set $U$ in $X$ containing $x$ such that $f(\mathrm{Cl}(U))$ is a subset of $\mathrm{Cl}(V) \quad(f(\mathrm{Cl}(U))$ is a subset of $V)$.

Evidently, each strongly $\theta$-continuous mapping is $\theta$-continuous.

Theorem 2.1. A $\theta$-continuous image of an almost Menger space is also almost Menger.

Proof. Let $X$ be an almost Menger space and $Y=f(X)$ its image under a $\theta$-continuous mapping $f: X \rightarrow Y$. Let $\left(\mathcal{V}_{n}\right)_{n \in N}$ be a sequence of open covers of $Y$. Fix $x \in X$. For each $n \in \boldsymbol{N}$ there is a set $V(x, n) \in \mathcal{V}_{n}$ containing $f(x)$. Since $f$ is $\theta$-continuous there is an open set $U(x, n)$ in $X$ containing $x$ such that $f(\mathrm{Cl}(U(x, n))$ is a subset of $\mathrm{Cl}(V(x, n))$. Therefore, for each $n$ the set $\mathcal{U}_{n}:=$ $\{U(x, n): x \in X\}$ is an open cover of $X$. As $X$ is almost Menger there is a sequence $\left(\mathcal{H}_{n}\right)_{n \in N}$ of finite sets such that for each $n, \mathcal{H}_{n}=\left\{U\left(x_{i}, n\right): i \leq k_{n}\right\}$ is a subset of $\mathcal{U}_{n}$ and $\quad \mathrm{X}=\bigcup_{n \in N} \mathrm{U}\left\{\mathrm{Cl}(H): H \in \mathcal{H}_{n}\right\}$. To each $U\left(x_{i}, n\right) \in \mathcal{H}_{n}$ assign a set $W\left(x_{i}, n\right) \in \mathcal{V}_{n}$ such that $f\left(\mathrm{Cl}\left(U\left(x_{i}, n\right)\right)\right.$ is a subset of $\mathrm{Cl}\left(W\left(x_{i}, n\right)\right)$ and set $\mathcal{W}_{n}=$ $\left\{W\left(x_{i}, n\right): i \leq k_{n}\right\}$. We obtain the sequence $\left(\mathcal{W}_{n}\right)_{n \in N}$ of finite subsets of $\mathcal{V}_{n}, n \in \boldsymbol{N}$, such that

$$
Y=f(X)=f\left(\bigcup_{n \in N} \bigcup\left\{\mathrm{Cl}(H): H \epsilon \mathscr{H}_{n}\right\}\right),
$$

which is by the previous a subset of

$$
\bigcup_{n \in N} \mathrm{U}\left\{\mathrm{Cl}(W): W \epsilon \mathcal{W}_{n}\right\}
$$

i.e. $Y$ is almost Menger.

Recall that a mapping $f: X \rightarrow Y$ is called almost continuous (Singal \& Singal, 1968) if the preimage of any regular open set $V$ in $Y$ is open in $X$. Each almost continuous mapping is $\theta$-continuous. So, the above theorem extends and generalizes the following result:

Corollary 2.2. (Kocev, 2009), Theorem 2.3 The almost Menger property is preserved by almost continuous mappings.

Similarly to the proof of Theorem 2.1 one proves the following theorem.

Theorem 2.3. A $\theta$-continuous image of an almost Rothberger space is also almost Rothberger.

Corollary 2.4. The almost Rothberger property is preserved by almost continuous mappings.

Definition 2.5. A space $X$ is said to be an almost Hurewicz space if for each sequence $\left(\mathcal{U}_{n}\right)_{n \in N}$ of open covers of $X$ there is a sequence $\left(\mathcal{V}_{n}\right)_{n \in N}$ such that for each $n, \mathcal{V}_{n}$ is a finite subset of $\mathcal{U}_{n}$ and for each $x \in X$, $x$ belongs to $\mathrm{Cl}\left(\mathrm{U} \mathcal{V}_{\mathrm{n}}\right)$ for all but finitely many $n$.

Theorem 2.6. $A \theta$-continuous image of an almost $\gamma$-set is an almost Hurewicz space.

Proof. Let $\left(\mathcal{V}_{n}\right)_{n \in N}$ be a sequence of open covers of $Y$. Let $x \epsilon X$. For each $n \in N$ there is $V(x, n) \in \mathcal{V}_{n}$ containing $f(x)$. Since $f$ is $\theta$-continuous there is an open set $U(x, n)$ in $X$ containing $x$ such that $\mathrm{f}(\mathrm{Cl}(U(x, n)))$ is a subset of $\mathrm{Cl}(V(x, n))$. For each $n$ let $\mathcal{U}_{\mathrm{n}}$ be the set of all finite unions of sets $U(x, n), x \in X$. Evidently, each $\mathcal{U}_{\mathrm{n}}$ is an $\omega$-cover of $X$. As $X$ is an almost $\gamma$-set there is a sequence $\left(U_{n}\right)_{n \in N}$ such that for each $n, U_{n} \in \mathcal{U}_{n}$ and each $x \in X$ does not belong to $\mathrm{Cl}\left(U_{n}\right)$ for at most finitely many $n$.

Let $\mathrm{U}_{\mathrm{n}}=\mathrm{U}\left(\mathrm{x}_{1}, \mathrm{n}\right) \mathrm{U} \mathrm{U}\left(\mathrm{x}_{2}, \mathrm{n}\right) \mathrm{U} \ldots \mathrm{U} \mathrm{U}\left(\mathrm{x}_{\mathrm{i}(\mathrm{n})}, \mathrm{n}\right)$. To each $U\left(x_{j}, n\right), \quad j \leq i(n)$, assign a set $V\left(x_{j}, n\right) \in \mathcal{V}_{n}$ with $\left.f(\mathrm{Cl}\}\left(U\left(x_{j}, n\right)\right)\right)$ a subset of $\mathrm{Cl}\left(V\left(x_{j}, n\right)\right)$. Let $y=f(x) \in Y$. Then from $x \in \mathrm{Cl}\left(U_{n}\right)$ for all $n \geq n_{0}$ for some $n_{0} \in N$, we get $x \in \mathrm{Cl}\left(U\left(x_{p}, n\right)\right)$ for some $1 \leq p \leq i(n)$ which implies $y \in f\left(\mathrm{Cl}\left(U\left(x_{p}, n\right)\right)\right)$ which is a subset of $\mathrm{Cl}\left(V\left(x_{p}, n\right)\right)$. If we put $\mathcal{W}_{n}=\left\{V\left(x_{j}, n\right): j=1,2, \ldots, i(n)\right\}$, we obtain the sequence $\left(\mathcal{W}_{n}\right)_{n \in N}$ of finite subsets of $\mathcal{V}_{n}, n \in N$, such that each $y \in Y$ belongs to all but finitely many sets $\mathrm{U}\left\{\mathrm{Cl}(W): W \in \mathcal{W}_{n}\right\}$. This just means that $Y$ is an almost Hurewicz space. $\square$

Theorem 2.7. A strongly $\theta$-continuous image $Y$ of an almost Menger space $X$ is a Menger space. 
Proof. Let $\left(\mathcal{V}_{n}\right)_{n \in N}$ be a sequence of open covers of $Y$. Let $x \in X$. For each $n \in \boldsymbol{N}$ there is a set $V_{x, n} \in \mathcal{V}_{n}$ containing $f(x)$. Since $f$ is strongly $\theta$-continuous there is an open set $U_{x, n}$ in $X$ containing $x$ such that $f\left(\mathrm{Cl}\left(U_{x, n}\right)\right.$ is a subset $V_{x, n}$. Therefore, for each $n$, the set $\mathcal{U}_{n}:=\left\{U_{x, n}: x \in X\right\}$ is an open cover of $X$. As $X$ is almost Menger there is a sequence $\left(\mathcal{F}_{n}\right)_{n \in N}$ of finite sets such that for each $n, \mathcal{F}_{n}$ is a subset of $\mathcal{U}_{n}$ and $X=$ $\bigcup_{n \in{ }_{N}} \mathrm{U}\left\{\mathrm{Cl}(F): F \in \mathcal{F}_{n}\right\}$.To each $F \in \mathcal{F}_{n}$ assign a set $W_{F} \in \mathcal{V}_{n}$ with $f(\mathrm{Cl}(F))$ a subset of $W_{F}$ and put $\mathcal{W}_{n}=$ $\left\{W_{F}: F \in \mathcal{F}_{n}\right\}$. We obtain the sequence $\left(\mathcal{W}_{n}\right)_{n \in N}$ of finite subsets of $\mathcal{V}_{n}, n \in N$, which witnesses for $\left(\mathcal{V}_{\mathrm{n}}\right)_{n \epsilon}$ ${ }_{N}$ that $Y$ is a Menger space, as it is easily checked.

In a quite similar way we prove the following theorem.

Theorem 2.8. A strongly $\theta$-continuous image $Y$ of an almost Hurewicz (almost Rothberger) space $X$ is a Hurewicz (Rothberger) space.

A mapping $f: X \rightarrow Y$ is called contra-continuous (Dontchev, 1996), if the preimage of each open set in $Y$ is closed in $X . \quad f$ is said to be precontinuous (Mashhour et al.,1982) if $f^{\leftarrow}(V)$ is a subset of $\mathrm{Int}(\mathrm{Cl}($ $\left.\left.f^{\leftarrow}(V)\right)\right)$ whenever $V$ is open in $Y$.

Theorem 2.9. A contra-continuous, precontinuous image $Y$ of an almost Menger space $X$ is a Menger space.

Proof. Let $\left(\mathcal{V}_{n}\right)_{n \in N}$ be a sequence of open covers of $Y$. Since $f$ is contra-continuous for each $n \in \mathbf{N}$ and each $V \epsilon \mathcal{V}_{n}$ the set $f^{\leftarrow}(V)$ is closed in $X$. On the other hand, because $f$ is precontinuous $f^{\leftarrow}(V)$ is a subset of $\operatorname{Int}\left(\mathrm{Cl}\left(f^{\leftarrow}(V)\right)\right)$, so that $f^{\leftarrow}(V)$ is a subset $\operatorname{Int}\left(f^{\leftarrow}(V)\right)$, i.e. $f^{\leftarrow}(V)=\operatorname{Int}\left(f^{\leftarrow}(V)\right)$. Therefore, for each $n$, the set $\mathcal{U}_{\mathrm{n}}=$ $\left\{f^{\leftarrow}(V): \mathrm{V} \epsilon \mathcal{V}\right\}$ is a cover of $X$ by open sets. As $X$ is an almost Menger space there is a sequence $\left(G_{n}\right)_{n \in \mathbf{N}}$ such that for each $n, G_{\mathrm{n}}$ is a finite subset of $\mathcal{U}_{n}$ and $\bigcup_{n \in N}$ $\mathrm{U}\left\{\mathrm{Cl}(\mathrm{G}): G \epsilon \mathcal{G}_{n}\right\}=X$. Then $\mathcal{W}_{n}=\left\{f(G): G \epsilon \mathcal{G}_{n}\right\}$ is a finite subset of $\mathcal{V}_{n}$ for each $n \in N$ and $\mathrm{U}_{n \in N} \cup \mathcal{W}_{n}$ is an open cover of $Y$. This means that $Y$ is a Menger space. $\square$

Recall that a mapping $f: X \rightarrow Y$ is called weakly continuous (Levine, 1960), (Levine, 1961), if for each $x \in X$ and each neighbourhood $V$ of $f(x)$ there is a neighbourhood $U$ of $x \operatorname{such} f(U)$ is contained in $\mathrm{Cl}(V)$.

Theorem 2.10. A weakly continuous image $Y$ of a Menger space $X$ is an almost Menger space.
Proof. Let $\left(\mathcal{V}_{n}\right)_{n \in N}$ be a sequence of open covers of $Y$. Let $x \in X$. Then for each $n \in N$ there is a $V \in \mathcal{V}_{n}$ such that $f(x) \in V$. Since $f$ is weakly continuous there is an open set $U_{x, n}$ in $X$ such that $x \in U_{x, n}$ and $f\left(U_{x, n}\right)$ is a subset of $\mathrm{Cl}(V)$. The set $\mathcal{U}_{n}:=\left\{U_{x, n}: x \in X\right\}$ is an open cover of $X$. Apply the fact that $X$ is a Menger space to the sequence $\left(\mathcal{U}_{n}\right)_{n \in N}$ and find a sequence $\left(\mathcal{F}_{n}\right)_{n \in N}$ of finite sets such that for each $n, \mathcal{F}_{\mathrm{n}}$ is a subset of $\mathcal{U}_{\mathrm{n}}$ and $\bigcup_{n \in N} \mathcal{F}_{\mathrm{n}}$ is an open cover of $X$. To each $n$ and each $U \epsilon \mathcal{F}_{n}$ assign a set $V_{U} \epsilon \mathcal{V}_{n}$ such that $f(U)$ is a subset of $\mathrm{Cl}\left(V_{U}\right)$ and put $\mathcal{W}_{\mathrm{n}}=\left\{\mathrm{V}_{\mathrm{U}}: \mathrm{U} \in \mathcal{F}_{\mathrm{n}}\right\}$. Then

$$
Y=f(X)=f\left(\bigcup_{n \in N} \bigcup\left\{U: U \in \mathcal{F}_{n}\right\}\right.
$$

which is contained in

$$
\bigcup_{n \in N} \bigcup\left\{\mathrm{Cl}\left(V_{U}\right): U \epsilon \mathcal{F}_{n}\right\},
$$

i.e. $Y$ is an almost Menger space. $\square$

\section{REFERENCES}

Babinkostova, L., Pansera, B. A., \& Scheepers, M. 2012. Weak covering properties and infinite games. Topology Appl., 159, pp. 3644-3657.

Babinkostova, L., Pansera, B. A., \& Scheepers, M. 2013. Weak covering properties and selection principles. Topology Appl., 160, pp. 2251-2271.

Daniels, P. 1988. Pixley-Roy spaces over subsets of the reals. Topology Appl., 29, pp. 93-106.

di Maio, G., \& Kocinac, D. R. Lj. 2008. Some covering properties of hyperspaces. Topology Appl., 155, pp. 1959-1969.

Dontchev, J. 1996. Contra-continuous functions and strongly S-closed spaces. Inter. J. Math. Math. Sci., 19, pp. 303-310.

Engelking, R. 1989. General Topology, 2nd ed.Berlin: Heldermann. Sigma Ser. Pure Math Vol. 6.

Fomin, S. V. 1941. Extensions of topological Spaces. Dokl. Acad. Nauk SSSR, 32, pp. 114-116.

Fomin, S. V. 1943. Extensions of topological spaces. Ann. Math., 44, pp. 471-480.

Gerlits, J., \& Zs. Nagy, 1982. Some properties of C(X) I. Topology Appl., 14, pp. 151-161.

Hurewicz, W. 1925. Uber eine Verallgemeinerung des Borelschen Theorems. Math. Z., 24, pp. 401-421.

Kocev, D. 2009. Almost Menger and related spaces. Mat. Vesnik, 61, pp. 173-180.

Kočinac, Lj. 1999. Star-Menger and related spaces II. Filomat, 13, pp. 129-140.

Kočinac, D. R. Lj. 2001. The Pixley-Roy topology and selection principles. Quest. Answers Gen. Topology, 19, pp. 219-225. 
Kočinac, D. R. Lj. 2006. Some covering properties in topological and uniform spaces. Proc. Steklov Inst. Math., 252, pp. 122-137.

Kočinac, D. R. Lj. 2015. Star selection principles: A survey. Khayyam Journal of Mathematics, 1, pp. 82106.

Levine, N. 1960. Strong continuity in topological spaces. Amer. Math. Monthly, 67, pp. 269-269.

Levine, N. 1961. A decpomposition of continuity in topolo gical spaces. Amer. Math. Monthly, 68, pp. 44-46.

Long, P. E., \& Herrington, L. L. 1981. Strongly $\theta$ continuous functions. J. Korean Math. Soc., 18, pp. 21-28.

Mashhour, A. S., El-Monsef, A. M. E., \& El-Deep, S.N. 1982. On precontinuous and weak precontinuous mappings.Proc. Math. Phys. Soc. Egypt, 53, pp. 47-53.

Menger, K. 1924. Einige Überdeckungssätze der Punktmengenlehre,

Sitzungsberichte. Math.
Astronomie, Physik, Meteorologie und Mechanik, 133, pp. 421-444. Abt. 2a.

Pansera, B. A. 2012. Weaker forms of the Menger property. Quaest. Math., 35, pp. 161-169.

Porter, J. R., \& Woods, R. G. 1988. Extensions and Absolutes of Hausdorff Spaces.Springer Verlag.

Rothberger, F. 1938. Eine Verschärfung der Eigenschalft C. Fund. Math., 30, pp. 50-55.

Sabah, A., ud Din Khan, M., \& Kočinac, D. R. Lj. 2016. Covering properties defined by semi-open sets. submitted.

Sakai, M. 2013. The weak Hurewicz property of Pixley-Roy hyperspaces. Topology Appl., 160, pp. 2531-2537.

Singal, M. K., \& Singal, A. R. 1968. Almost continuous mappings. Yokohama Math. J., 16, pp. 63-73.

Willard, S., \& Dissanayake, U. N. B. 1984. The almost Lindelöf degree. Canadian Math. Bull., 27, pp. 452-455.

\footnotetext{
*E-mail: 1kocinac@gmail.com
} 